\title{
Investigation of high-order cell-centered finite difference method for aeroacoustics
}

\author{
Yao Jin ${ }^{*}$ \\ National Key Laboratory of Aerodynamic Design and Research, School of Aeronautics, Northwestern Polytechnical \\ University, Xi'an, 710072, P.R. China \\ Fei $\operatorname{Liao}^{\dagger}$ \\ State Key Laboratory of Nonlinear Mechanics, Institute of Mechanics, China Academy of Science, Beijing, 100190 , \\ P.R. China \\ Jinsheng Cai ${ }^{\ddagger}$ \\ National Key Laboratory of Aerodynamic Design and Research, School of Aeronautics, Northwestern Polytechnical \\ University, Xi'an, 710072, P.R. China \\ and \\ Philip J. Morris $\S$ \\ Department of Aerospace Engineering, the Pennsylvania State University, University Park, PA, 16802, USA
}

\begin{abstract}
This paper discusses the potential of the application of a high-order cell-centered finite difference method (CCFDM) to problems in aeroacoustics. Based on an in-house code, a class of low-dissipation and low-dispersion compact schemes adopting optimization techniques is proposed for direct noise calculation of long-time long-distance sound propagation problems. These schemes are further validated with benchmark cases from a CAA workshop and the results indicate these schemes outperform the widely-used 5th-order upwind scheme. The second part of this paper investigates the capability of combining CCFDM with the FW-H acoustic analogy for the far-field noise prediction. Two example cases, including a tonal noise case (laminar flow past a 2D NACA0012 airfoil) and a broadband case (turbulent flow past a 3D rod-airfoil configuration), are both analyzed in detail. Good agreement with experimental data has been achieved, which indicates that the CCFDM coupled with the FW-H acoustic analogy has great potential in aeroacoustics.
\end{abstract}

\section{Introduction}

Computational Aeroacoustics (CAA) is a topic of considerable interest to the computational fluid dynamics (CFD) community. In terms of the differences between acoustic perturbations and flow perturbations, on one hand, acoustic fluctuations are usually small in the magnitude. On the other hand, acoustic fluctuations can propagate for long distances with nearly unnoticeable damping. Such properties limit the conventional CFD numerical tools in CAA. One obvious shortcoming in conventional CFD methods is the excessive truncation error resulting from the low-order numerical methods which will overwhelm the acoustic signals. Hence, many high-order low-dissipation and low-dispersion schemes have been proposed ${ }^{1,2}$, particularly for aeroacoustic problems.

However, even though these high-order schemes have been successfully applied in turbulence and jet noise, when it comes to complicated configurations with poor grid quality, numerical errors arising from the complexity of the grid are still a problem. One reason is that numerical methods for the curvilinear Navier-Stokes (N-S) equations violate the geometric conservative law $(\mathrm{GCL})^{3}$. To remedy this, Deng et al. ${ }^{4}$ proposed a new method for spatial

\footnotetext{
* Ph.D student, Department of Fluid Mechanics. Email address: jinyao@mail.nwpu.edu.cn.

$\dagger$ Postdoc researcher, State Key Laboratory of Nonlinear Mechanics. Email address: liaofei@imech.ac.cn.

* Corresponding author. Professor, Department of Fluid Mechanics, AIAA Fellow. Email address: caijsh@nwpu.edu.cn.

$\S$ Boeing/A.D. Welliver Professor, Aerospace Engineering, AIAA fellow. Email address: pjm@psu.edu.
} 
discretization and a new method for geometric treatment (called SCMM), which makes it possible to adopt high-order finite difference method for complicated geometric configurations ${ }^{5}$. Later, Liao et al. ${ }^{6,7}$ noticed the connection between a cell-centered finite volume method and Deng's node-centered finite difference method. Deng's method ${ }^{4}$ has been further extended to be a cell-centered finite difference method (CCFDM), along with the conservative cell-centered SCMM (CCSCMM) to satisfy the GCL. Liao's method ${ }^{6}$ provides a better physical explanation from the perspective of conservation, especially at the multiblock interfaces.

It has been shown that the interpolation scheme plays a dominate role in spectral property ${ }^{8}$. Therefore, developing compact interpolation schemes to improve the spectral performance has begun to draw researchers' attention. A class of linear dissipative compact interpolations ${ }^{9}$ has been proposed. These schemes have inherent dissipation compared with non-dissipative central compact interpolations. In the present paper, new compact interpolation schemes will be proposed where both the dispersion property and dissipation property are improved dramatically.

An efficient and accurate way to extrapolate the noise signals in source region to the far-field region is called a wave extrapolation method ${ }^{10}$. For the near-field source region, the accuracy is strongly associated with the CFD tools, which is the reason why low-dissipation and low-dispersion schemes are the primary interest in CCFDM. For the far-field radiation process, a commonly used method involves the solution of the FW-H equation ${ }^{10}$ which is an exact rearrangement of N-S equations into a wave propagation equation. There are two classical strategies to obtain the numerical solutions to the $\mathrm{FW}-\mathrm{H}$ equations: the time domain formulation ${ }^{11,12}$ and the frequency domain formulation ${ }^{13,14}$. The time domain formulation is obtained based on the 3D Green function. The frequency domain formulation can be used for both 2D and 3D problems depending on whether the 2D or 3D Green function is adopted. In this paper, the convective effect is also included. As illustrations, the tonal noise radiation from laminar flow past a 2-D NACA0012 airfoil ${ }^{15}$ is investigated to indicate that the CCFDM combined with frequency domain FW-H formulation is effective. In addition, a broadband noise prediction from the 3 -D rod-airfoil configuration ${ }^{16}$ is simulated and compared with the experimental data to demonstrate the use of the method.

The organization of this paper is as follows. In section II, the general methodology, CCFDM coupled with CCSCMM, is introduced. For direct noise calculations, a class of low-dissipation low-dispersion interpolations within the framework of CCFDM is proposed. For far-field noise prediction, the wave extrapolation method (FW-H equation) is reintroduced where the convective effect is also included. Both the time and frequency domain formulations are given. Section III provides benchmark cases for validation. One category is to validate the developed low-dissipation low-dispersion interpolations for direct noise calculation. The other category demonstrates the feasibility of combining CCFDM with the FW-H equations for far-field noise predictions. Conclusions are provided in Section IV.

\section{Governing Equations and Methodologies}

\section{A. A cell-centered finite difference method (CCFDM)}

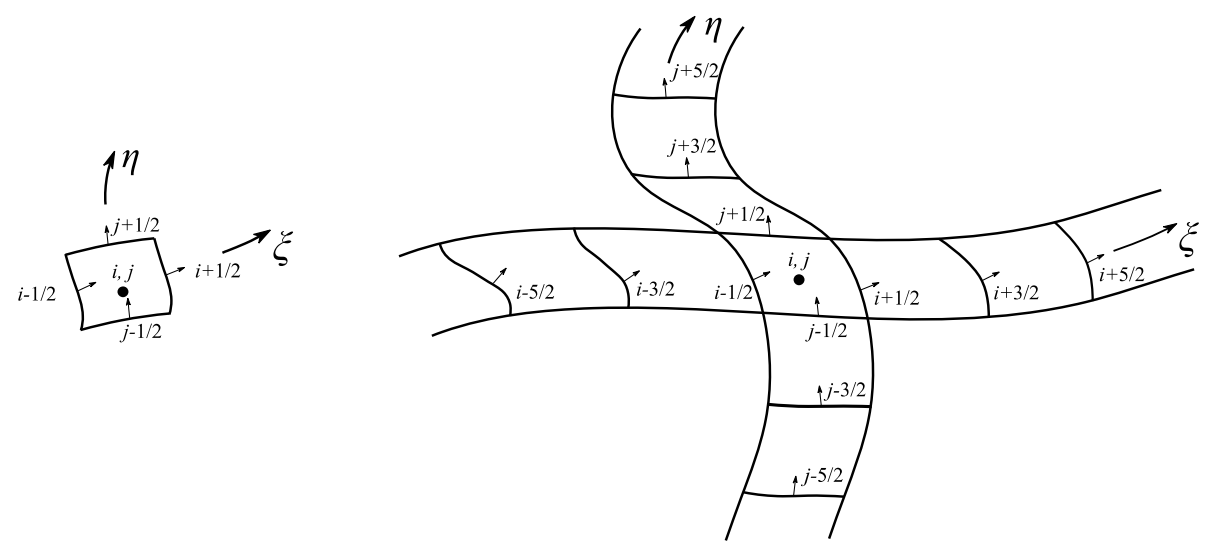

Figure 1. Comparison between the FVM and CCFDM in spatial discretization.

The non-dimensional Navier-Stokes (N-S) equations in the curvilinear coordinates are 


$$
\frac{\partial \boldsymbol{U}(\boldsymbol{Q})}{\partial t}+\frac{\partial \boldsymbol{E}(\boldsymbol{Q})}{\partial \xi}+\frac{\partial \boldsymbol{F}(\boldsymbol{Q})}{\partial \eta}+\frac{\partial \boldsymbol{G}(\boldsymbol{Q})}{\partial \zeta}=\frac{M a}{R e}\left(\frac{\partial \boldsymbol{E}_{v}(\boldsymbol{Q})}{\partial \xi}+\frac{\partial \boldsymbol{F}_{v}(\boldsymbol{Q})}{\partial \eta}+\frac{\partial \boldsymbol{G}_{v}(\boldsymbol{Q})}{\partial \zeta}\right),
$$

where details can be seen in Reference $e^{6,7}$.

For simplicity, a one-dimensional scalar equation is used as a demonstration. The semi-discrete form is written as,

$$
\frac{d Q}{d t}=-\left.\frac{\partial E(Q)}{\partial \xi}\right|_{i}
$$

where $\partial E / \partial \xi$ is discretized with compact difference schemes ${ }^{17}$

$$
\begin{gathered}
\left.\beta \frac{\partial E}{\partial \xi}\right|_{i-2}+\left.\alpha \frac{\partial E}{\partial \xi}\right|_{i-1}+\left.\frac{\partial E}{\partial \xi}\right|_{i}+\left.\alpha \frac{\partial E}{\partial \xi}\right|_{i+1}+\left.\beta \frac{\partial E}{\partial \xi}\right|_{i+2}= \\
a\left(E_{i+1 / 2}-E_{i-1 / 2}\right)+\frac{b}{2}\left(E_{i+1}-E_{i-1}\right)+\frac{c}{3}\left(E_{i+3 / 2}-E_{i-3 / 2}\right)+\frac{d}{4}\left(E_{i+2}-E_{i-2}\right)+\frac{e}{5}\left(E_{i+5 / 2}-E_{i-5 / 2}\right)
\end{gathered},
$$

where the fluxes on face centers are calculated with a Riemann flux solver which is given by,

$$
E_{i+1 / 2}=E\left(Q_{i+1 / 2}^{L}, Q_{i+1 / 2}^{R}, J \xi_{x, i+1 / 2}, J \xi_{y, i+1 / 2}, J \xi_{z, i+1 / 2}\right),
$$

where the left and right interpolated primitive variables on the $i+1 / 2$ face center $Q_{i+1 / 2}^{L}$ and $Q_{i+1 / 2}^{R}$ should be obtained using high-order schemes. A discussion of coordinate transformation metrics, indicated by $J \xi_{x, i+1 / 2}$, $J \xi_{y, i+1 / 2}$ and $J \xi_{z, i+1 / 2}$ are given in section B. Figure 1 shows a comparison between the diagrammatic sketch of stencils used in a 2nd-order Finite Volume Method (FVM) and a high-order CCFDM.

\section{B. Cell-centered symmetric conservative metric method (CCSCMM)}

The cell-centered symmetric conservative metric method (CCSCMM $)^{6,7}$ is designed to discretize the surface metrics and Jacobians so as to satisfy the Geometric Conservation Law (GCL). The symmetric conservative calculations for surface metrics $J \xi_{x, i+1 / 2}, J \xi_{y, i+1 / 2}$ and $J \xi_{z, i+1 / 2}$ are given by

$$
\begin{aligned}
& \left(J \xi_{x}\right)^{S 3}=\frac{1}{2}\left[\left(J \xi_{x}\right)^{S 1}+\left(J \xi_{x}\right)^{S 2}\right],\left(J \xi_{y}\right)^{S 3}=\frac{1}{2}\left[\left(J \xi_{y}\right)^{S 1}+\left(J \xi_{y}\right)^{S 2}\right],\left(J \xi_{z}\right)^{S 3}=\frac{1}{2}\left[\left(J \xi_{z}\right)^{S 1}+\left(J \xi_{z}\right)^{S 2}\right], \\
& \left(J \eta_{x}\right)^{S 3}=\frac{1}{2}\left[\left(J \eta_{x}\right)^{S 1}+\left(J \eta_{x}\right)^{S 2}\right],\left(J \eta_{y}\right)^{S 3}=\frac{1}{2}\left[\left(J \eta_{y}\right)^{S 1}+\left(J \eta_{y}\right)^{S 2}\right],\left(J \eta_{z}\right)^{S 3}=\frac{1}{2}\left[\left(J \eta_{z}\right)^{S 1}+\left(J \eta_{z}\right)^{S 2}\right], \\
& \left(J \zeta_{x}\right)^{S 3}=\frac{1}{2}\left[\left(J \zeta_{x}\right)^{S 1}+\left(J \zeta_{x}\right)^{S 2}\right],\left(J \zeta_{y}\right)^{S 3}=\frac{1}{2}\left[\left(J \zeta_{y}\right)^{S 1}+\left(J \zeta_{y}\right)^{S 2}\right],\left(J \zeta_{z}\right)^{S 3}=\frac{1}{2}\left[\left(J \zeta_{z}\right)^{S 1}+\left(J \zeta_{z}\right)^{S 2}\right],
\end{aligned}
$$

where

$$
\begin{aligned}
& \left(J \xi_{x}\right)^{S 1}=\left(y_{\eta} z\right)_{\zeta}-\left(y_{\zeta} z\right)_{\eta},\left(J \xi_{y}\right)^{S 1}=\left(z_{\eta} x\right)_{\zeta}-\left(z_{\zeta} x\right)_{\eta},\left(J \xi_{z}\right)^{S 1}=\left(x_{\eta} y\right)_{\zeta}-\left(x_{\zeta} y\right)_{\eta}, \\
& \left(J \eta_{x}\right)^{S 1}=\left(y_{\zeta} z\right)_{\xi}-\left(y_{\xi} z\right)_{\zeta},\left(J \eta_{y}\right)^{S 1}=\left(z_{\zeta} x\right)_{\xi}-\left(z_{\xi} x\right)_{\zeta},\left(J \eta_{z}\right)^{S 1}=\left(x_{\zeta} y\right)_{\xi}-\left(x_{\xi} y\right)_{\zeta}, \\
& \left(J \zeta_{x}\right)^{S 1}=\left(y_{\xi} z\right)_{\eta}-\left(y_{\eta} z\right)_{\xi},\left(J \zeta_{y}\right)^{S 1}=\left(z_{\xi} x\right)_{\eta}-\left(z_{\eta} x\right)_{\xi},\left(J \zeta_{z}\right)^{S 1}=\left(x_{\xi} y\right)_{\eta}-\left(x_{\eta} y\right)_{\xi}, \\
& \left(J \xi_{x}\right)^{S 2}=\left(y z_{\zeta}\right)_{\eta}-\left(y z_{\eta}\right)_{\zeta},\left(J \xi_{y}\right)^{S 2}=\left(z x_{\zeta}\right)_{\eta}-\left(z x_{\eta}\right)_{\zeta},\left(J \xi_{z}\right)^{S 2}=\left(x y_{\zeta}\right)_{\eta}-\left(x y_{\eta}\right)_{\zeta}, \\
& \left(J \eta_{x}\right)^{S 2}=\left(y z_{\xi}\right)_{\zeta}-\left(y z_{\zeta}\right)_{\xi},\left(J \eta_{y}\right)^{S 2}=\left(z x_{\xi}\right)_{\zeta}-\left(z x_{\zeta}\right)_{\xi},\left(J \eta_{z}\right)^{S 2}=\left(x y_{\xi}\right)_{\zeta}-\left(x y_{\zeta}\right)_{\xi}, \\
& \left(J \zeta_{x}\right)^{S 2}=\left(y z_{\eta}\right)_{\zeta}-\left(y z_{\xi}\right)_{\eta},\left(J \zeta_{y}\right)^{S 2}=\left(z x_{\eta}\right)_{\xi}-\left(z x_{\xi}\right)_{\eta},\left(J \zeta_{z}\right)^{S 2}=\left(x y_{\eta}\right)_{\xi}-\left(x y_{\xi}\right)_{\eta} .
\end{aligned}
$$

The above equations are discretized numerically, as an illustration, in the following manner,

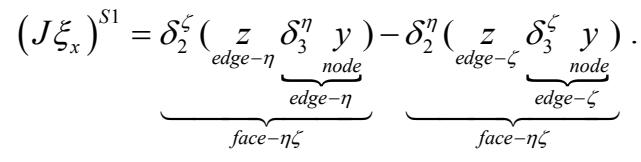


Given the difference operator given by Eq.(3) as $\delta_{1}$, the following necessary condition should be satisfied to meet GCL requirements.

$$
\delta_{1}=\delta_{2}=\delta_{3} .
$$

The Jacobian in Eq.(5) and Eq.(6) is calculated by

$$
\begin{aligned}
& J=\frac{1}{3}\{\underbrace{\delta_{1}^{\xi}[\underbrace{x}_{\text {face }-\eta \zeta \zeta} \underbrace{\left(J \xi_{x}\right)}_{\text {face }-\eta \zeta}+\underbrace{y}_{\text {face }-\eta \zeta} \underbrace{\left(\xi_{\text {face }} \eta \zeta \zeta\right.}_{\text {face }-\left(J \xi_{y}\right)} \underbrace{\left(J \xi_{z}\right)}_{\text {face }-\eta \zeta}]}_{\text {cell- }-\xi \eta \zeta}
\end{aligned}
$$

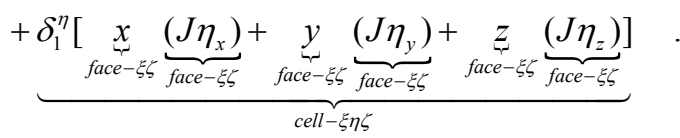

$$
\begin{aligned}
& +\underbrace{\delta_{1}^{\zeta}[\underbrace{x}_{\text {face }-\xi \eta \eta} \underbrace{\left(J \zeta_{x}\right)}_{\text {face }-\xi \eta}+\underbrace{y}_{\text {face }-\xi \eta}(\underbrace{\left(J \zeta_{y}\right)}_{\text {face }}+\underbrace{z}_{\text {face }-\xi \eta} \underbrace{\left(J \zeta_{z}\right)}_{\text {face }-\xi \eta}]}_{\text {cell }-\xi \eta \xi}\}
\end{aligned}
$$

Based on above theoretical developments, an in-house parallel structured compressible solver has been developed by Liao ${ }^{6}$ and all the following results in the present paper are based on this in-house solver.

\section{Low-dissipation and low-dispersion compact interpolations}

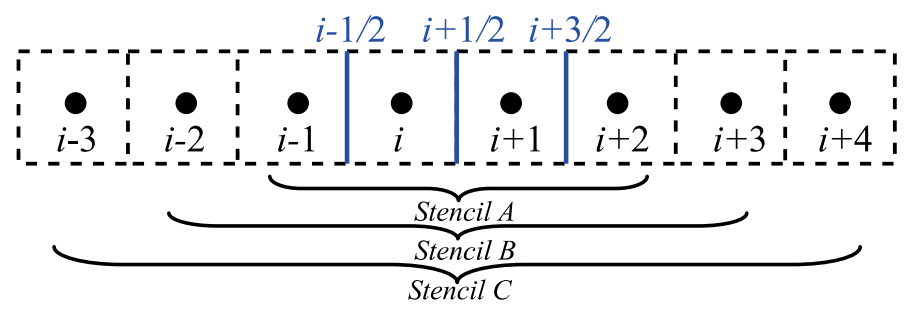

Figure 2. Schematic diagram of the stencil for compact interpolations.

Figure 2 depicts a schematic diagram of the stencil for compact interpolations, which is given as

$$
\beta_{-1} Q_{i-\frac{1}{2}}+Q_{i+\frac{1}{2}}+\beta_{1} Q_{i+\frac{3}{2}}=c_{-3} Q_{i-3}+c_{-2} Q_{i-2}+c_{-1} Q_{i-1}+c_{0} Q_{i}+c_{1} Q_{i+1}+c_{2} Q_{i+2}+c_{3} Q_{i+3}+c_{4} Q_{i+4},
$$

where the coefficients are listed in Table 1.

Table 1. Coefficients of schemes in Eq. (10) with various order.

\begin{tabular}{ccccccc}
\hline & $\beta_{-1}, \beta_{1}$ & $c_{-3}, c_{4}$ & $c_{-2}, c_{3}$ & $c_{-1}, c_{2}$ & $c_{0}, c_{1}$ & $\begin{array}{c}\text { Transfer function } \\
T(k)\end{array}$ \\
\hline Opt4 & $\frac{\eta \mp \xi}{2}$ & 0 & 0 & $\frac{-1+3 \eta \mp 2 \xi}{16}$ & $\frac{9+5 \eta \mp 10 \xi}{16}$ & Eq.(11) \\
\hline Opt6 & $\frac{\eta \mp \xi}{2}$ & 0 & $\frac{3-5 \eta \pm 2 \xi}{256}$ & $\frac{-25+63 \eta \mp 42 \xi}{256}$ & $\frac{5(15+7 \eta \mp 14 \xi)}{128}$ & Eq.(12) \\
\hline Opt8 & $\frac{\eta \mp \xi}{2}$ & $\frac{-5+7 \eta \mp 2 \xi}{2048}$ & $\frac{49-75 \eta \pm 30 \xi}{2048}$ & $\frac{7(-35+81 \eta \mp 54 \xi)}{2048}$ & $\frac{175(7+3 \eta \mp 6 \xi)}{2048}$ & Eq.(13) \\
\hline
\end{tabular}

The transfer function for Opt4 is

$$
T(k)_{O p t 4}=\frac{\left(\frac{1}{8}(-1+3 \eta) \cos \left(\frac{3}{2} k\right)+\frac{1}{8}(9+5 \eta) \cos \left(\frac{1}{2} k\right)\right)+i\left(\frac{1}{4} \xi \sin \left(\frac{3}{2} k\right)+\frac{5}{4} \xi \sin \left(\frac{1}{2} k\right)\right)}{(\eta \cos (k)+1)+i(\xi \sin (k))} .
$$

The transfer function for Opt6 is 


$$
T(k)_{O p t 6}=\frac{\left(\frac{1}{128}(3-5 \eta) \cos \left(\frac{5}{2} k\right)+\frac{1}{128}(-25+63 \eta) \cos \left(\frac{3}{2} k\right)+\frac{5}{64}(15+7 \eta) \cos \left(\frac{1}{2} k\right)\right)}{+i\left(-\frac{1}{64} \xi \sin \left(\frac{5}{2} k\right)+\frac{21}{64} \xi \sin \left(\frac{3}{2} k\right)+\frac{35}{32} \xi \sin \left(\frac{1}{2} k\right)\right)}-.
$$

The transfer function for Opt8 is

$$
T(k)_{\text {Opt }}=\frac{\left(\begin{array}{l}
\frac{1}{1024}(-5+7 \eta) \cos \left(\frac{7}{2} k\right)+\frac{1}{1024}(49-75 \eta) \cos \left(\frac{5}{2} k\right)+ \\
\frac{1}{1024}(-245+567 \eta) \cos \left(\frac{3}{2} k\right)+\frac{1}{1024}(1225+525 \eta) \cos \left(\frac{1}{2} k\right)
\end{array}\right)+i\left(\begin{array}{l}
\frac{1}{512} \xi \sin \left(\frac{7}{2} k\right)-\frac{15}{512} \xi \sin \left(\frac{5}{2} k\right)+ \\
\frac{189}{512} \xi \sin \left(\frac{3}{2} k\right)+\frac{525}{512} \xi \sin \left(\frac{1}{2} k\right)
\end{array}\right)}{(\eta \cos (k)+1)+i(\xi \sin (k))} .
$$

The real part $T_{\mathfrak{R}}(k)$ and imaginary part $T_{\mathfrak{Y}}(k)$ of transfer function $T(k)$ affect the dispersion and dissipation respectively. The recommended $\xi$ and $\eta$ are defined by an optimization criterion, which is given below. Some of the subjective parameters are specified by experience and trial and error.

(1) The following integrated error function should reach its minimum value.

$$
E=\int_{0}^{k_{c}}\left\{\sigma\left|T_{\Re}(k)-1\right|+(1-\sigma)\left|T_{\Im}(k)-0\right|\right\} d k,
$$

where $k_{c}$ is the cut-off wavenumber below which the optimization procedure is valid. $\sigma$ is a weight between 0 and 1 . In this paper, $\sigma$ is chosen to be 0.5 .

(2) The amount of built-in dissipation should be as small as possible under the condition that no noticeable numerical oscillations be observed in all the benchmark cases.

(3) Dispersive error, denoted as $\left|T_{\mathfrak{R}}(k)-1\right|$, should be controlled within an appropriate constraint. After trials, $2 \%, 1 \%$ and $0.5 \%$ are recommended for Opt 4 , Opt 6 and Opt 8 respectively.

Finally, the optimized values of $\xi$ and $\eta$ for Opt4, Opt6 and Opt8 are given in Table 2. Furthermore, the spectral properties are shown and compared with a 5th-order upwind explicit scheme (UE5) and a 5th-order upwind compact scheme (UI5) in Figure 3. It can be seen that Opt4, Opt6 and Opt8 improve the spectral properties dramatically.

Table 2 The optimized $\xi$ and $\eta$ for Opt4, Opt6 and Opt8.

\begin{tabular}{cccc}
\hline & Opt4 & Opt6 & Opt8 \\
\hline$\eta$ & 0.731 & 0.810 & 0.848 \\
\hline$\xi$ & -0.104 & -0.080 & -0.062 \\
\hline
\end{tabular}

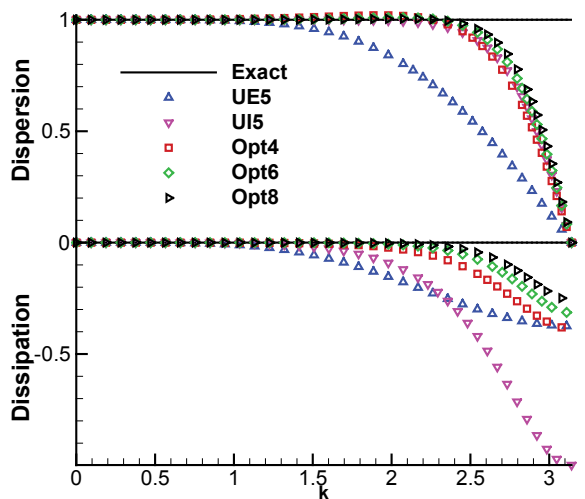

(a) Interpolations

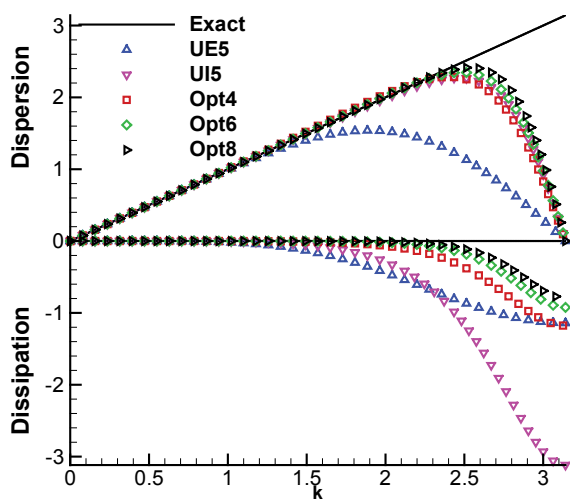

(b) Interpolations combined with difference schemes

Figure 3. Spectral property comparison.

\section{Ffowes Williams and Hawkings (FW-H) equation}

The convective $\mathrm{FW}-\mathrm{H}$ equation ${ }^{12}$ is given as follows 


$$
\left(\frac{D^{2}}{c^{2} D t^{2}}-\nabla^{2}\right)\left(p^{\prime} H(f)\right)=\frac{\partial^{2}\left(T_{i j} H(f)\right)}{\partial x_{i} \partial x_{j}}-\frac{\partial}{\partial x_{i}}\left(L_{i j} n_{j} \delta(f)\right)+\frac{D}{D t}\left(\rho_{0} U_{i} n_{i} \delta(f)\right),
$$

where

$$
\begin{aligned}
& \frac{D}{D t}=\frac{\partial}{\partial t}+U_{\infty, i} \frac{\partial}{\partial x}, \\
& T_{i j}=\rho u_{i} u_{j}+P_{i j}-c^{2} \rho^{\prime} \delta_{i j}, \\
& L_{i j}=\rho u_{i}\left(u_{j}-\left(v_{j}-U_{\infty, j}\right)\right)+P_{i j}, \\
& U_{i}=\left(v_{i}-U_{\infty, i}\right)+\rho\left(u_{i}-\left(v_{i}-U_{\infty, i}\right)\right) / \rho_{0} .
\end{aligned}
$$

Eq.(15) can be solved in both the time domain ${ }^{11,12}$ and the frequency domain ${ }^{13,14}$.

\section{D.1 Time domain formulation}

The time domain solution ${ }^{11,12}$ for Eq.(15), where the contribution of volume integration is ignored, is written as

$$
4 \pi p^{\prime}\left(\mathbf{x}, t, \vec{M}_{\infty}\right)=\int_{f=0}\left[\left(1-M_{\infty, i} R_{i}\right) \frac{\rho_{0} \dot{U}_{i} n_{i}}{R^{*}}-c M_{\infty, i} R_{i}^{*} \frac{U_{i} n_{i}}{R^{* 2}}\right]_{r e t} d S+\int_{f=0}\left[\frac{1}{c} \frac{\dot{L}_{i j} n_{j} R_{i}}{R^{*}}+\frac{L_{i j} n_{j} R_{i}^{*}}{R^{*}}\right]_{r e t} d S,
$$

where,

$$
\begin{aligned}
& R_{i}=\gamma^{2}\left(-M_{\infty, i}+R_{i}^{*}\right), \\
& R=\gamma^{2}\left(R^{*}-\vec{M}_{\infty} \cdot(\mathbf{x}-\mathbf{y})\right), \\
& R_{i}^{*}=\frac{\left(x_{i}-y_{i}\right)+\gamma^{2}\left(M_{\infty, j} \cdot\left(x_{j}-y_{j}\right)\right) M_{\infty, i}}{\gamma^{2} R^{*}}, \\
& R^{*}=\frac{1}{\gamma} \sqrt{(\mathbf{x}-\mathbf{y})^{2}+\gamma^{2}\left(\vec{M}_{\infty} \cdot(\mathbf{x}-\mathbf{y})\right)^{2}},
\end{aligned}
$$

and where $\gamma^{2}=1 /\left(1-\left|\vec{M}_{\infty}\right|^{2}\right)$. The dot above $\dot{L}_{i j}, \dot{U}_{i}$ means a source time derivative. $[\bullet]_{\text {ret }}$ indicates that the integration is implemented at the retarded source time $\tau$, which can be obtained by

$$
t=\tau+|\mathbf{x}-\mathbf{y}(\tau)| / c .
$$

Detailed descriptions of above terms can be found in Reference ${ }^{12}$.

\section{D.2 Frequency domain formulation}

The frequency domain solution ${ }^{13,14}$ for Eq.(15), where the contribution of volume integration is ignored, is given as

$$
p^{\prime}(\mathbf{x}, \omega) H(f)=-\int_{S} F(\mathbf{y}, \omega) \frac{\partial G(\mathbf{x}, \mathbf{y}, \omega)}{\partial y_{i}} d S-\int_{S} i \omega Q(\mathbf{y}, \omega) G(\mathbf{x}, \mathbf{y}, \omega) d S .
$$

Whether 2D or 3D problem is concerned depends on the choice of Green function $G(\mathbf{x}, \mathbf{y}, \omega)$.

The 3D Green function in the frequency domain and its derivatives are

$$
\begin{aligned}
& G(\boldsymbol{x}, \boldsymbol{y}, \omega)=\frac{1}{4 \pi S} e^{-i k\left(-M_{r}+S\right) / \beta^{2}}, \\
& \frac{\partial G(\boldsymbol{x}, \boldsymbol{y}, \omega)}{\partial y_{i}}=\frac{1}{4 \pi S} e^{-i k\left(-M_{r}+S\right) / \beta^{2}}\left\{-\frac{1}{S^{2}}\left(-\beta^{2} r_{i}-M_{i} M_{r}\right)-\frac{i k}{\beta^{2}}\left(M_{i}+\frac{-\beta^{2} r_{i}-M_{i} M_{r}}{S}\right)\right\},
\end{aligned}
$$

where $S=\sqrt{\beta^{2} \vec{r}^{2}+M_{r}^{2}}, M_{r}=\vec{M}_{\infty} \cdot \vec{r}, \vec{r}=\vec{x}-\vec{y}, \quad \beta^{2}=1-\left|\vec{M}_{\infty}\right|^{2}$.

The 2D Green function in the frequency domain and its derivatives are given by 


$$
\begin{aligned}
& G(\boldsymbol{x}, \boldsymbol{y}, \omega)=\frac{i}{4 \beta} \exp \left(\frac{i k M \bar{r}_{1}}{\beta^{2}}\right) H_{0}^{(2)}\left(\frac{k}{\beta^{2}} S\right) \\
& \frac{\partial G(\boldsymbol{x}, \boldsymbol{y}, \omega)}{\partial y_{1}}=\exp \left(\frac{i M k \overline{r_{1}}}{\beta^{2}}\right)\left\{\frac{k M \cos \theta}{4 \beta^{3}} H_{0}^{(2)}\left(\frac{k}{\beta^{2}} S\right)-\frac{i k\left(\beta^{2} \sin \theta \overline{r_{2}}-\cos \theta \overline{r_{1}}\right)}{4 \beta^{3} S} H_{1}^{(2)}\left(\frac{k}{\beta^{2}} S\right)\right\} \\
& \frac{\partial G(\boldsymbol{x}, \boldsymbol{y}, \omega)}{\partial y_{2}}=\exp \left(\frac{i M k \bar{r}_{1}}{\beta^{2}}\right)\left\{\frac{k M \sin \theta}{4 \beta^{3}} H_{0}^{(2)}\left(\frac{k}{\beta^{2}} S\right)-\frac{i k\left(-\beta^{2} \cos \theta \bar{r}_{2}-\sin \theta \bar{r}_{1}\right)}{4 \beta^{3} S} H_{1}^{(2)}\left(\frac{k}{\beta^{2}} S\right)\right\}
\end{aligned}
$$

where $S=\sqrt{\bar{r}_{1}^{2}+\beta^{2} \bar{r}_{2}^{2}}, \quad \bar{r}_{1}=r_{1} \cos \theta+r_{2} \sin \theta \quad, \quad \bar{r}_{2}=-r_{1} \sin \theta+r_{2} \cos \theta \quad, \quad r_{i}=x_{i}-y_{i}, i=1,2 . \quad \beta^{2}=1-|M|^{2}$, $M=\sqrt{U^{2}+V^{2}} / c . \quad \theta=\tan ^{-1}(V / U)$ represents the angle of attack.

Detailed descriptions of above terms can be found in Reference ${ }^{14}$.

\section{Numerical validations}

\section{A. Convective isentropic vortex}

This problem describes a $2 \mathrm{D}$ moving isentropic vortex located at the center of the computational domain $\left(x_{c}, y_{c}\right)=(0,0)$ moving in the $x$ direction with the Mach number 0.5 . The initial velocity, temperature and entropy fluctuations are as follows,

$$
\begin{aligned}
& (\delta u, \delta v)=\frac{\beta}{2 \pi} e^{\left(\frac{1-r^{2}}{2}\right)}\left[-\left(y-y_{c}\right),\left(x-x_{c}\right)\right], \delta T=-\frac{(\gamma-1) \beta^{2}}{8 \gamma \pi^{2}} e^{\left(1-r^{2}\right)}, \rho=T^{\frac{1}{\gamma-1}}, \\
& P=\rho T, r=\sqrt{\left(x-x_{c}\right)^{2}+\left(y-y_{c}\right)^{2}},
\end{aligned}
$$

where the vortex strength $\beta$ is 5 . The simulation ends at $t=100$. The performance of various schemes in preserving the strength of the vortex after a long-time simulation is investigated. Figure 4 depicts the density profiles. It can be seen that UI5 exhibits dramatic improvement over UE5. Moreover, the optimized schemes behave less dissipatively than UI5.

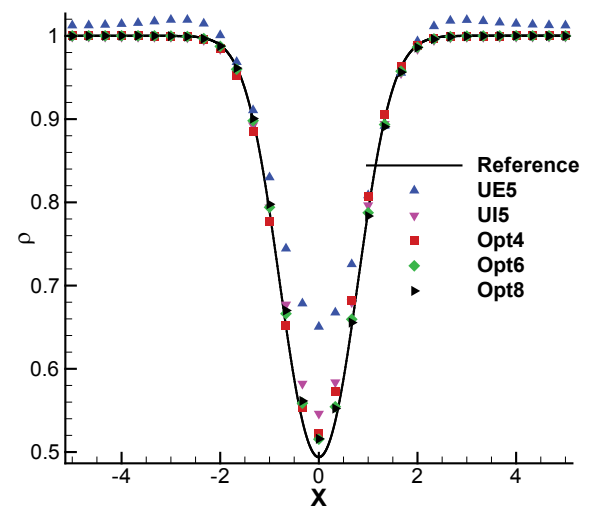

(a) On grid with $30 \times 30$ cell centers

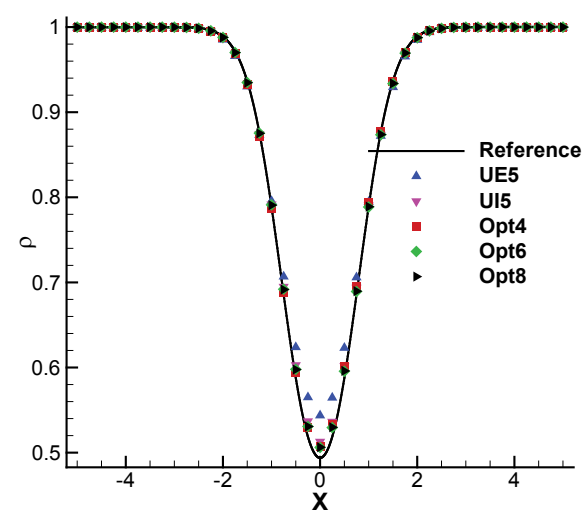

(b) On grid with $40 \times 40$ cell centers

Figure 4. Density profile of convective isentropic vortex.

\section{B. 2D-pulse-entropy-vorticity propagation}

This problem ${ }^{18}$ consists of an acoustic wave, a vorticity wave and an entropy wave propagating in a uniform flow with Mach number 0.5. The initial perturbation variables are given as 


$$
\left\{\begin{array}{l}
p^{\prime}=\varepsilon \exp \left[-(\ln 2)\left(x^{2}+y^{2}\right) / 9\right] \\
\rho^{\prime}=\varepsilon \exp \left[-(\ln 2)\left(x^{2}+y^{2}\right) / 9\right]+0.1 \varepsilon \exp \left[-(\ln 2)\left((x-67)^{2}+y^{2}\right) / 25\right] \\
u^{\prime}=0.04 \varepsilon y \exp \left[-(\ln 2)\left((x-67)^{2}+y^{2}\right) / 25\right] \\
v^{\prime}=-0.04 \varepsilon(x-67) \exp \left[-(\ln 2)\left((x-67)^{2}+y^{2}\right) / 25\right]
\end{array}\right.
$$

where $\varepsilon=0.001$. This is because, for nonlinear Euler equations, a sufficiently small source strength has to be introduced. Correspondingly, the numerical results need to multiply by 1000 so as to match the analytical solution. Similar post-processing is taken for case $C$ and D. The computational domain is $[-100,100] \times[-100,100]$ on a uniform grid with $\Delta x=\Delta y=2$.

Figure 5 shows density fluctuation contours and density line graphs along the central line at three different times. It is clear that UE5 produces noticeable dispersive and dissipative error. By contrast, the dissipation error is eliminated with UI5 but the numerical dispersive error has still not been improved. Opt6 and Opt8 give solutions closest to the exact solutions.
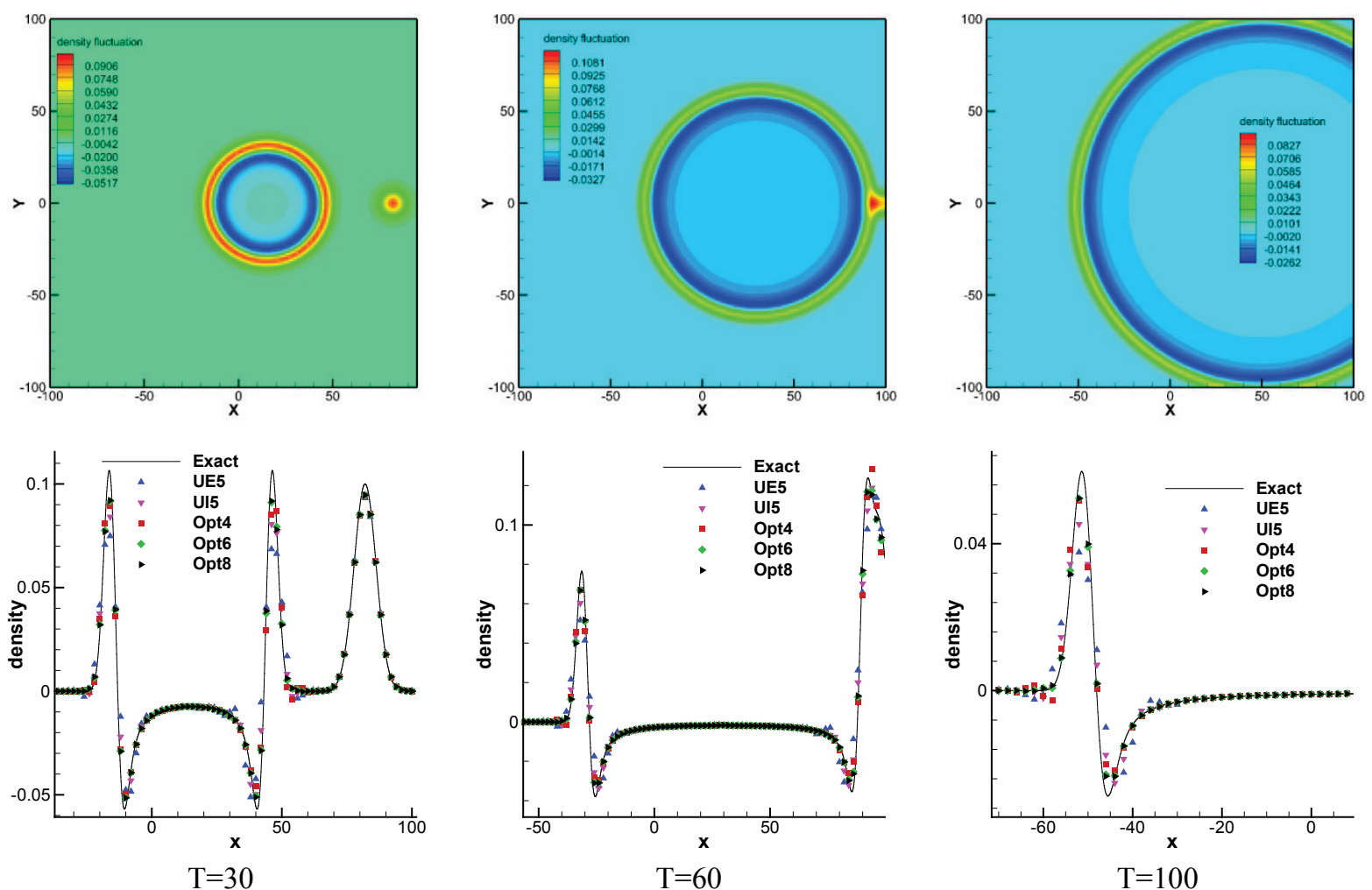

Figure 5. Density contours (above) and density profiles along the central line (below) for pulse-entropy-vorticity propagation problem.

\section{Scattering of a sound wave from a solid wall}

This problem ${ }^{18}$ illustrates how the acoustic wave propagates in the uniform flow with $M a=0.5$ and interacts with a solid wall. The computational domain is $[-100,100] \times[0,200]$. The bottom boundary is treated as a solid wall. The initial perturbations are

$$
\left\{\begin{array}{l}
p^{\prime}=\varepsilon \exp \left[-(\ln 2)\left(x^{2}+(y-25)^{2}\right) / 25\right] \\
\rho^{\prime}=\varepsilon \exp \left[-(\ln 2)\left(x^{2}+(y-25)^{2}\right) / 25\right] \\
u^{\prime}=v^{\prime}=0
\end{array}\right.
$$


where $\varepsilon=0.001$. The grid is uniformly spaced with $\Delta x=\Delta y=2$. Figure 6 shows density perturbation contours and line graphs at three different times along the dashed line. Compared with UE5 which generates marginal dispersive error, the results produced by the compact schemes agree well with the analytical solutions.
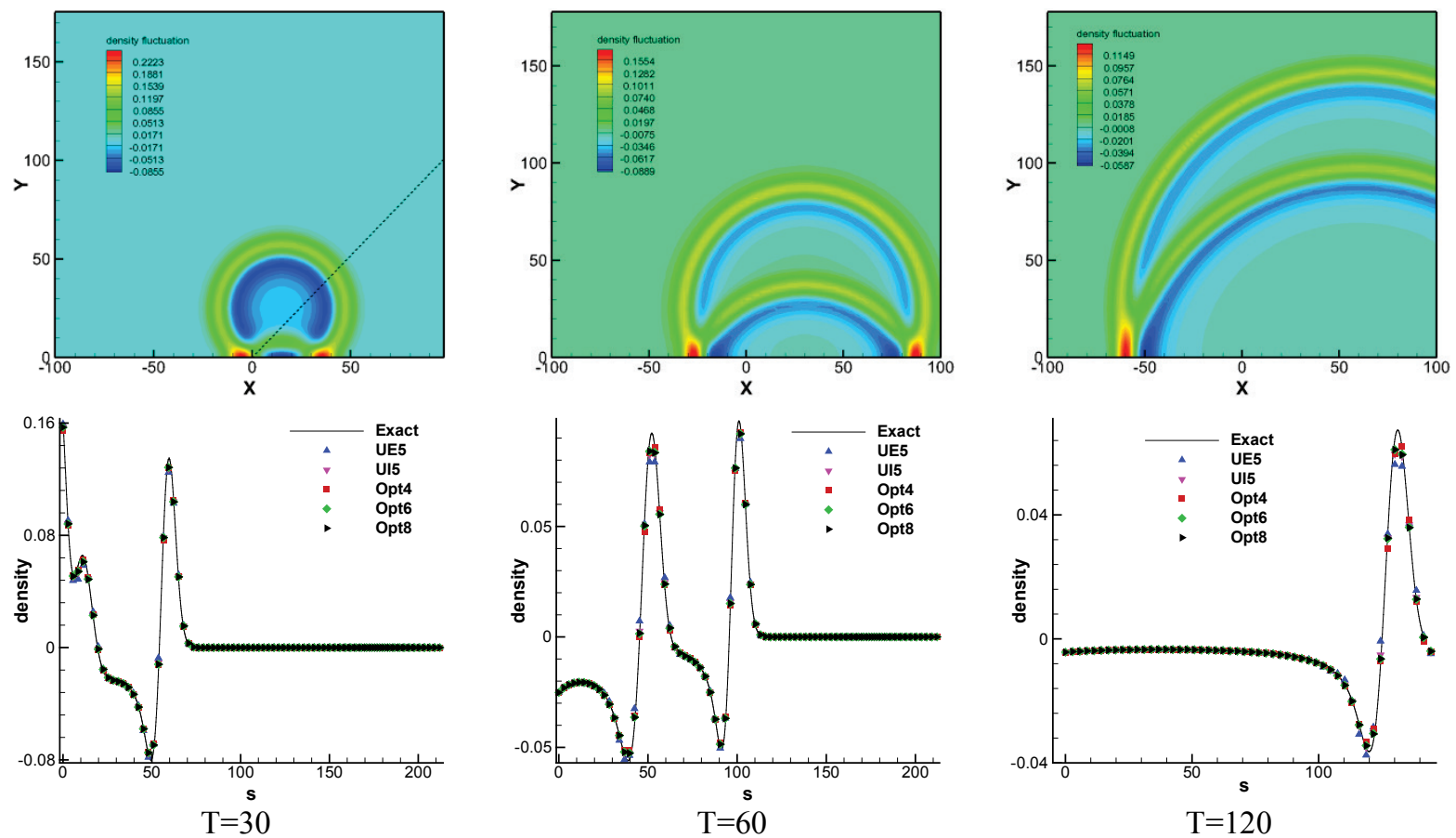

Figure 6. Density contours (above) and density profiles along the dashed line (below) for sound radiation from wall.

\section{Scattering of sound wave from multi-cylinder}

This case ${ }^{19}$ is to further investigate the sound radiation from complex configurations. A periodic source is added to the energy equation in Eq.(1).

$$
S=\varepsilon \exp \left[-25(\ln 2)\left(x^{2}+y^{2}\right)\right] \sin (8 \pi t),
$$

where $\varepsilon=0.001$. Both two and three-cylinder cases are investigated. The grid for the two and three-cylinder cases are spaced with $\Delta x \approx \Delta y \approx 0.035$ and $\Delta x \approx \Delta y \approx 0.035$ respectively.

Figure 7 show the instantaneous pressure perturbations and root-mean-square (RMS) pressure contours. The waves radiated from the cylinders are captured. To further investigate the capability of different schemes, the calculated results are compared with the exact solution in Figure 8. It is observed that the line with UE5 deviates from the exact solution. By contrast, UI5 shows a dramatic improvement. Furthermore, the optimized schemes (Opt4, Opt6 and Opt8) give additional improvements over UI5.
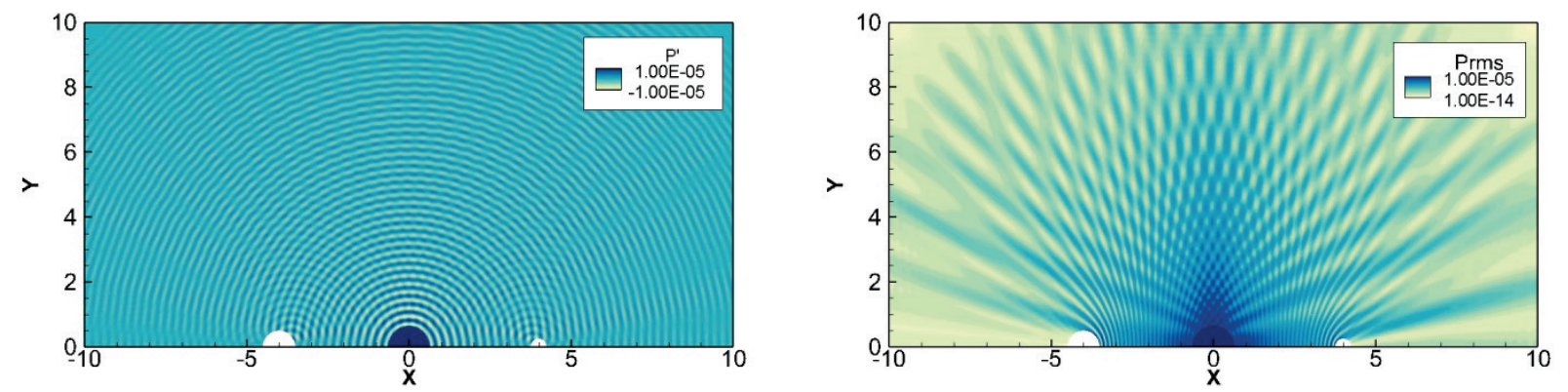

(a) Two cylinders 

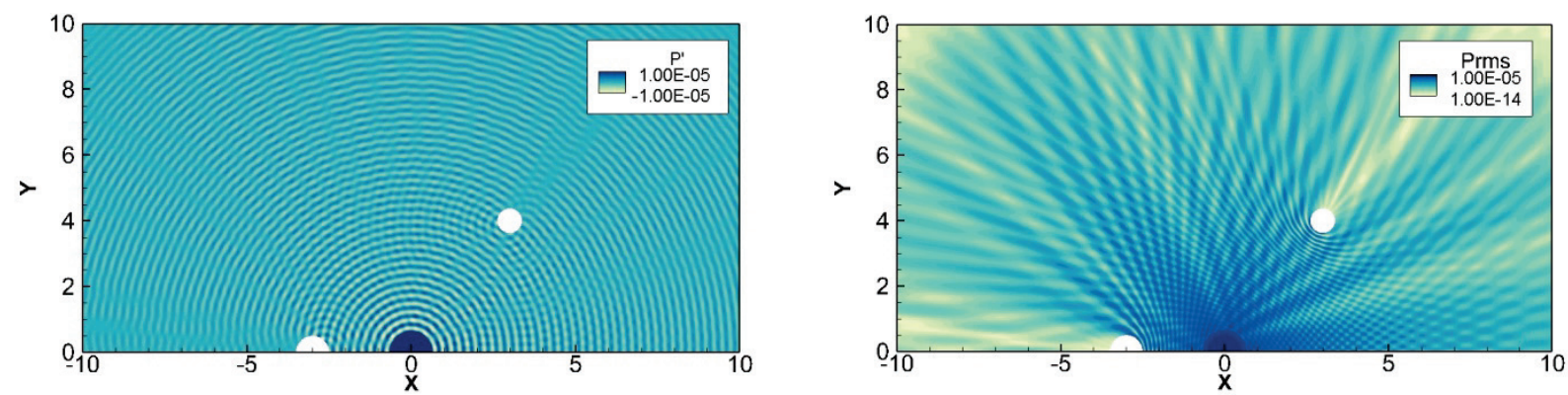

(b) Three cylinders

Figure 7. Pressure fluctuation contours. Left: instantaneous contours; Right: RMS contours.

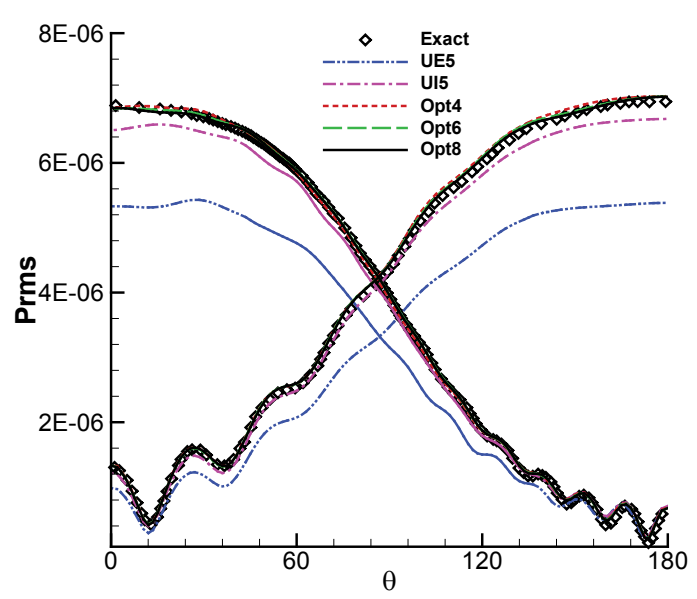

(a) Two cylinders.

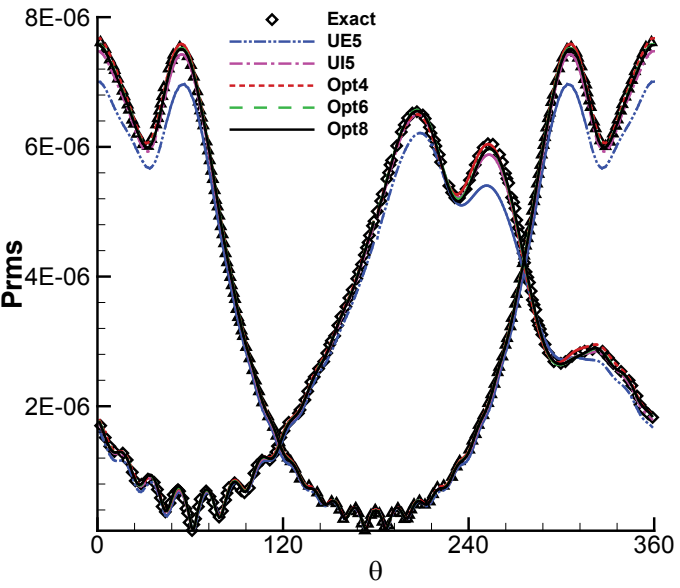

(b) Three cylinders.

Figure 8. Comparison of RMS pressure fluctuations on the surface of cylinders.

\section{E. Tonal noise radiated from the 2D NACA0012 airfoil}

The Reynolds number based on the NACA0012 airfoil chord is $R e=5000$ and the Mach number is given as 0.3 . The angle of attack is 5 degree. The observer points are located on a circle with radius $17 c$. Figure 9 illustrates the periodic vortex shedding from the trailing edge of the NACA0012 airfoil.

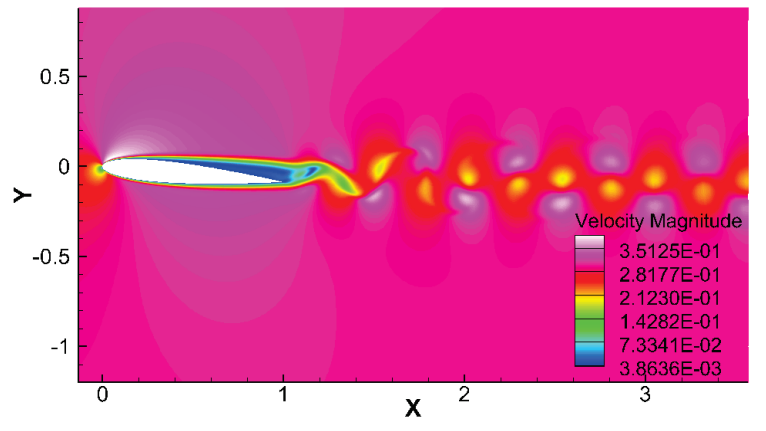

Figure 9. Instantaneous velocity magnitude contour of NACA 0012 at the 5 degree angle of attack. 


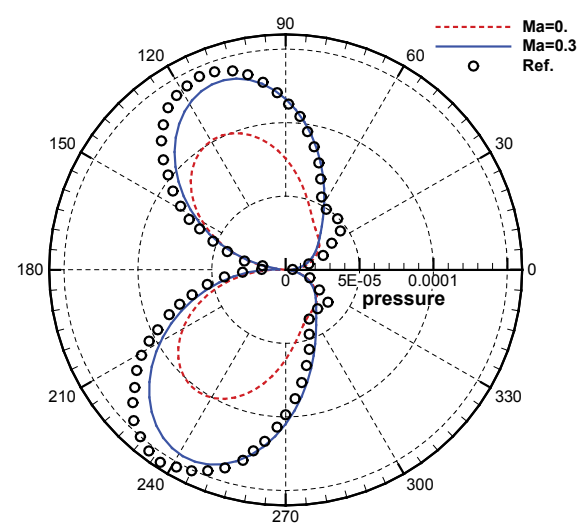

Figure 10. Farfield pressure fluctuation directivity at $R=17 c$.

The frequency-domain formulation for the FW-H equation is solved to obtain the far-field sound directivity. Input data is collected on the surface of airfoil. The calculated DNS data ${ }^{15}$ is included for reference. Figure 10 indicates that the convective effect has a noticeable influence on the far-field directivity pattern. The small discrepancy between the DNS data and the present results stems from the neglect of the volume integrations. Even so, this case still shows that the CCFDM combined with the frequency-domain formulation can give satisfactory result for $2 \mathrm{D}$ problems.

\section{F. Broadband noise radiated from the 3D rod-airfoil configuration}

This case ${ }^{16}$ has been used as a benchmark case for broadband airframe noise. The rod with $d=0.01 \mathrm{~m}$ is placed upstream of the symmetric NACA 0012 airfoil with chord $c=0.1 \mathrm{~m}$. The distance between the rod and the airfoil is $1 \mathrm{~m}$. The incoming velocity is $72 \mathrm{~m} / \mathrm{s}$, corresponding to $M a=0.2$. The Reynolds number based on the rod diameter and the airfoil chord are $R e_{d}=4.8 \times 10^{4}$ and $R e_{c}=4.8 \times 10^{5}$ respectively.

The computational domain extends from $-8 c$ to $16 c$ in the streamwise direction and from $-8 c$ to $8 c$ in the cross-stream direction. Additionally, the spanwise length is chosen to be $0.3 c$. Figure 11 shows details of the grid on the 2D central spanwise plane and the total cells approximate 0.16 million. The spanwise direction is divided into 44 cells, which gives rise to about 7 million total cells for the whole CFD computational domain.
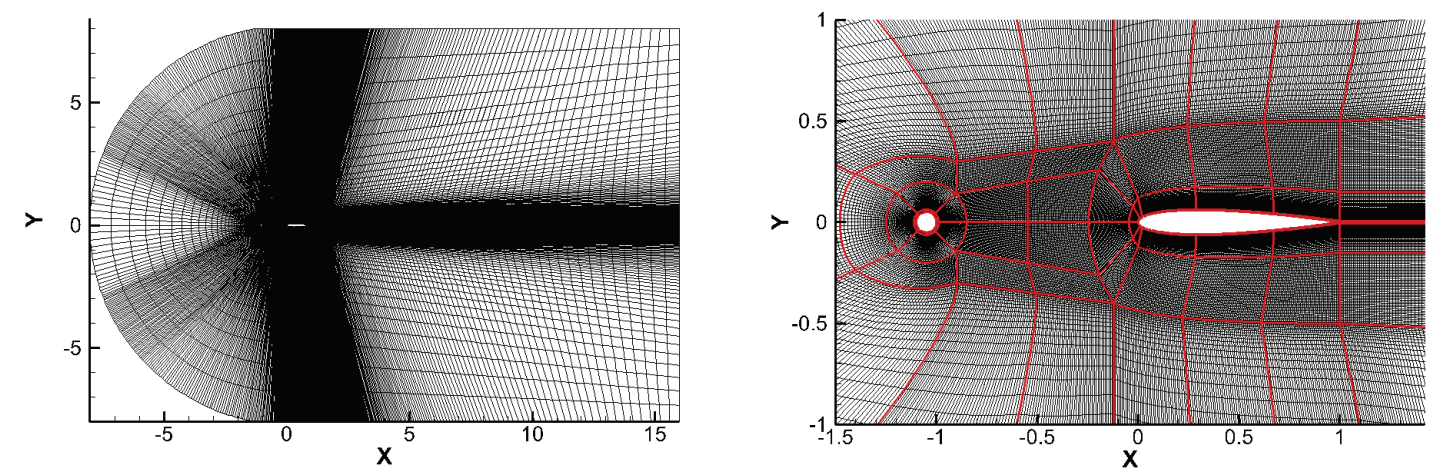

Figure 11. View of the grid on the central spanwise plane. Left: global. Right: zoom in on a closer configuration.

The numerical strategy for spatial discretization is based on the optimized 6th-order monotonicity-preserving scheme $^{20}$ and the time-marching step is the iteratively implicit DDADI method ${ }^{21}$, which accelerates the convergence rate remarkably by the large allowable CFL number. The turbulence model is the Delayed Detached Eddy Simulation $^{22}$. The non-dimensional numerical time step is 0.03 . The CFD simulation ends when statistical convergence has been achieved. 


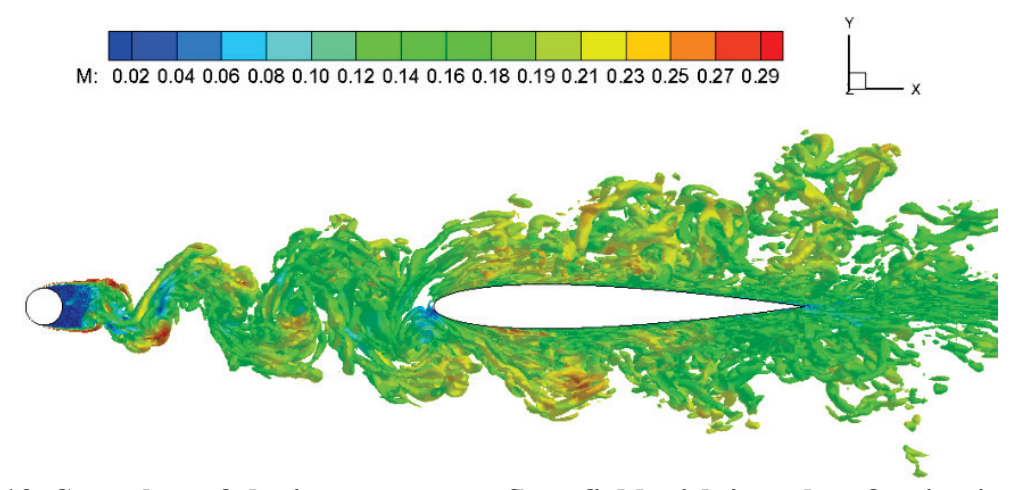

Figure 12. Snapshot of the instantaneous flow-field with isovalue $Q$-criterion $(Q=2)$.

Figure 12 gives an overview of the instantaneous flow-field, where the turbulent wake shedding from the rod is clearly observed and these turbulent structures impact on the leading edge of the airfoil. The calculated surface pressure coefficient on the rod is compared in Figure 13 with the experimental data from Apelt et al. ${ }^{23}$. The pressure distribution on the airfoil is compared with LES data from Giert et $\mathrm{al}^{24}$.
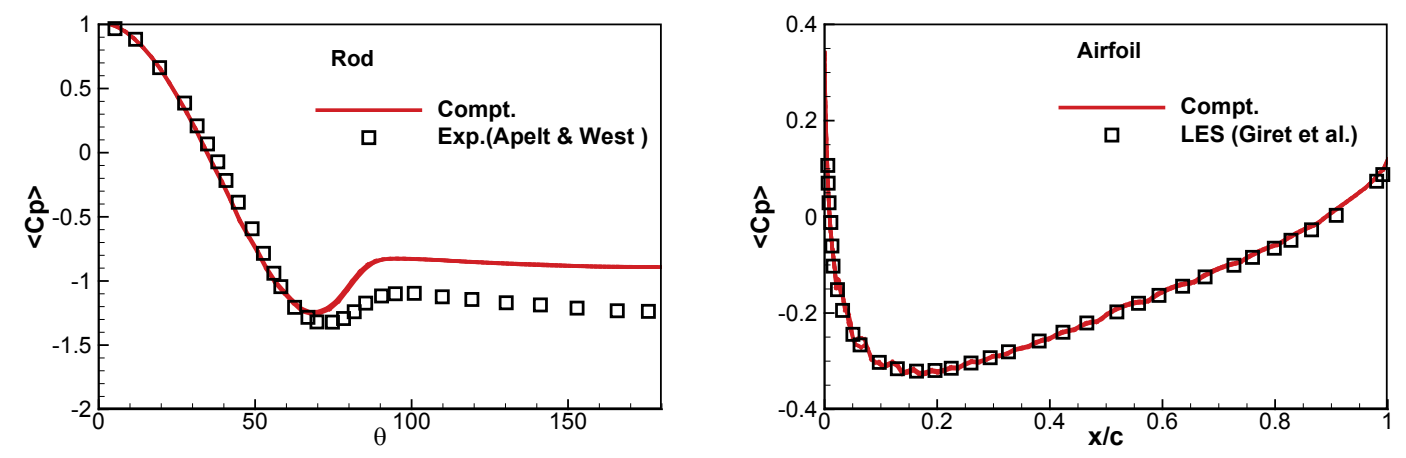

Figure 13. Mean surface pressure coefficient on the rod (left) and airfoil (right).

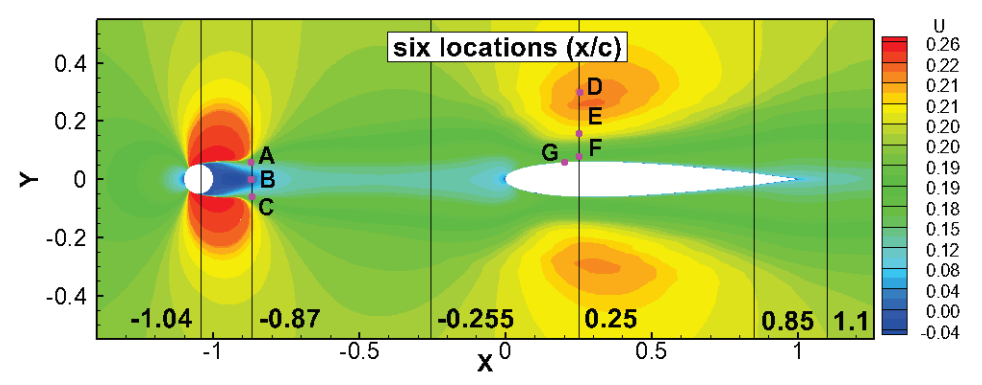

Figure 14. Mean streamwise velocity contour. The locations for the mean velocity (Figure 15) and RMS velocity (Figure 16) fluctuation profiles are sketched by solid black lines. The power spectral density (PSD) of streamwise velocity fluctuations (Figure 17) are collected at 6 locations (A,B,C and D,E,F). The PSD of unsteady pressure fluctuations (Figure 18) is collected at point $G$ on the upper surface of the airfoil.

Figure 14 shows the mean streamwise velocity contour. The near region velocity profiles at six streamwise locations are further compared with the experimental data in Figure 15 and Figure 16. Noticeably, except for the results at $x / c=-0.87$, the numerical solutions agree well with the experimental data, though the measured data at $x / c=1.1$ behaves with a slight asymmetric pattern. This asymmetry has been clarified by Jacob et al. ${ }^{16}$ that the rod in the experiment is slightly misaligned with the airfoil by about $2 \mathrm{~mm}$ in the vertical direction. Additionally, as for the discrepancy between experimental data and calculated results at $x / c=-0.87$, Agrawal et al ${ }^{25}$ describe concerns about the measured data and they point out that the peak velocity deficit in the wake is expected to reduce with the distance away from the rod. 

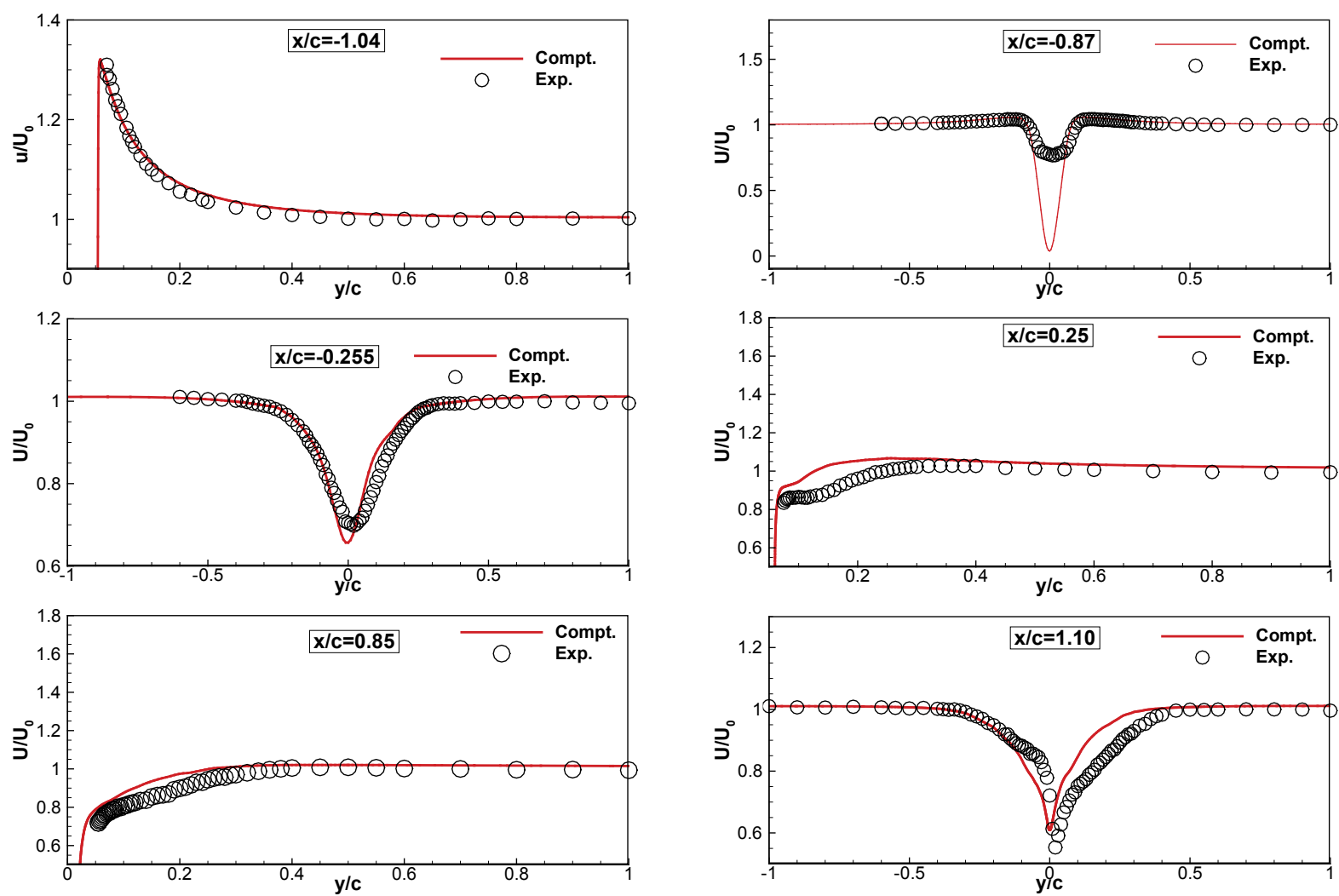

Figure 15. Mean streamwise velocity profiles $u / U_{0}$ at various streamwise locations.
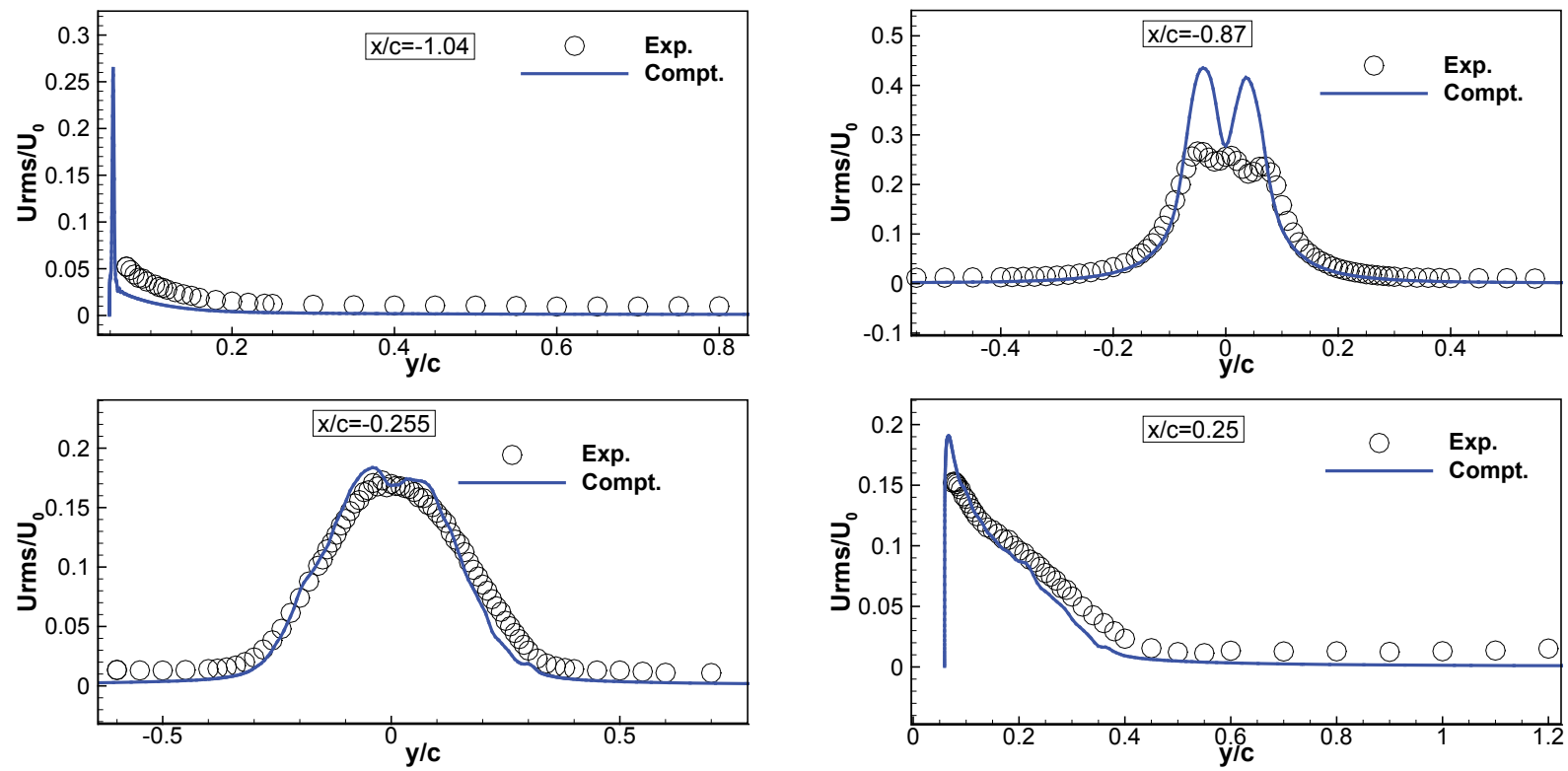

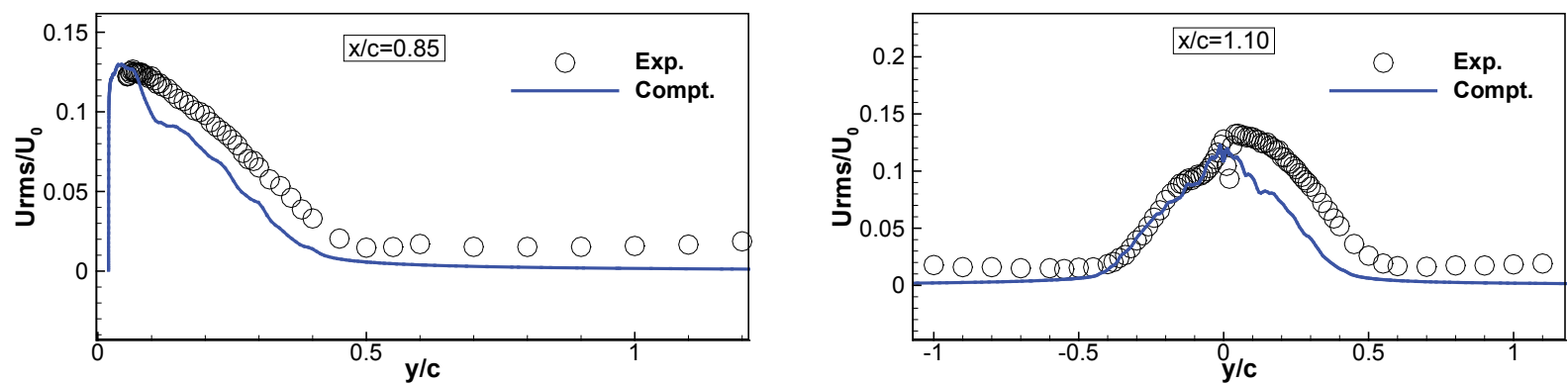

Figure 16. RMS streamwise fluctuation velocity profiles $\sqrt{\overline{u^{\prime} u^{\prime}}} / U_{0}$ at various streamwise locations.

The PSD of streamwise velocity fluctuations at the specific locations (A,B,C and D,E,F shown in Figure 14) are shown in Figure 17. The fundamental tonal fluctuation due to vortex shedding from the rod is clearly resolved at point $\mathrm{A}$ and point $\mathrm{C}$. Results at points $\mathrm{D}, \mathrm{E}$ and $\mathrm{F}$ indicate the vortices shed from the rod still dominant the velocity field surrounding the airfoil. Due to the lack of grid resolution, the prediction at point $\mathrm{D}$ is lower than the measured data whilst the calculated data for the nearest point $\mathrm{F}$ collapses well with the experimental data. Figure 18 also shows the surface pressure PSD at location G, which also demonstrates the good agreement with the experimental data.
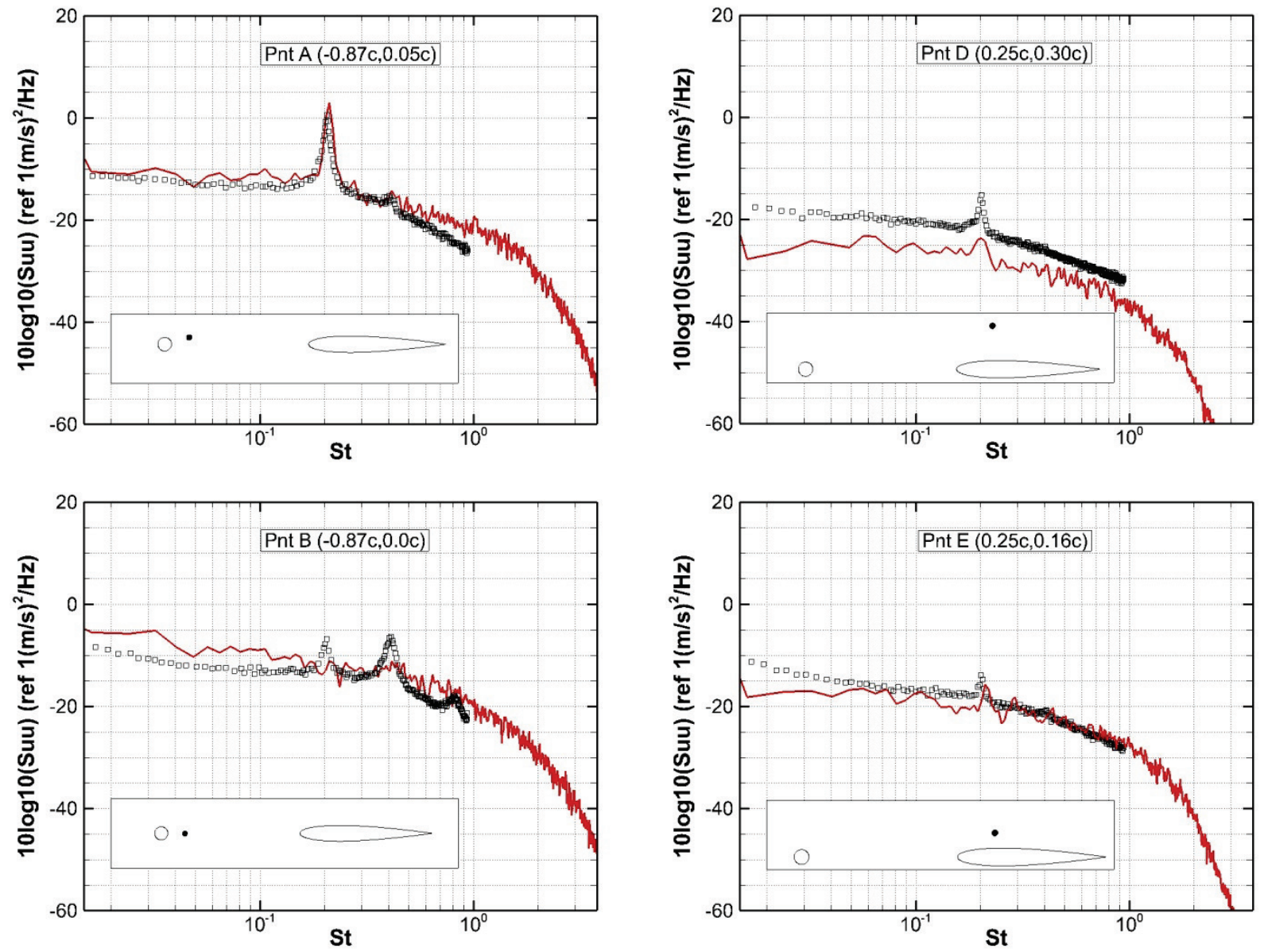

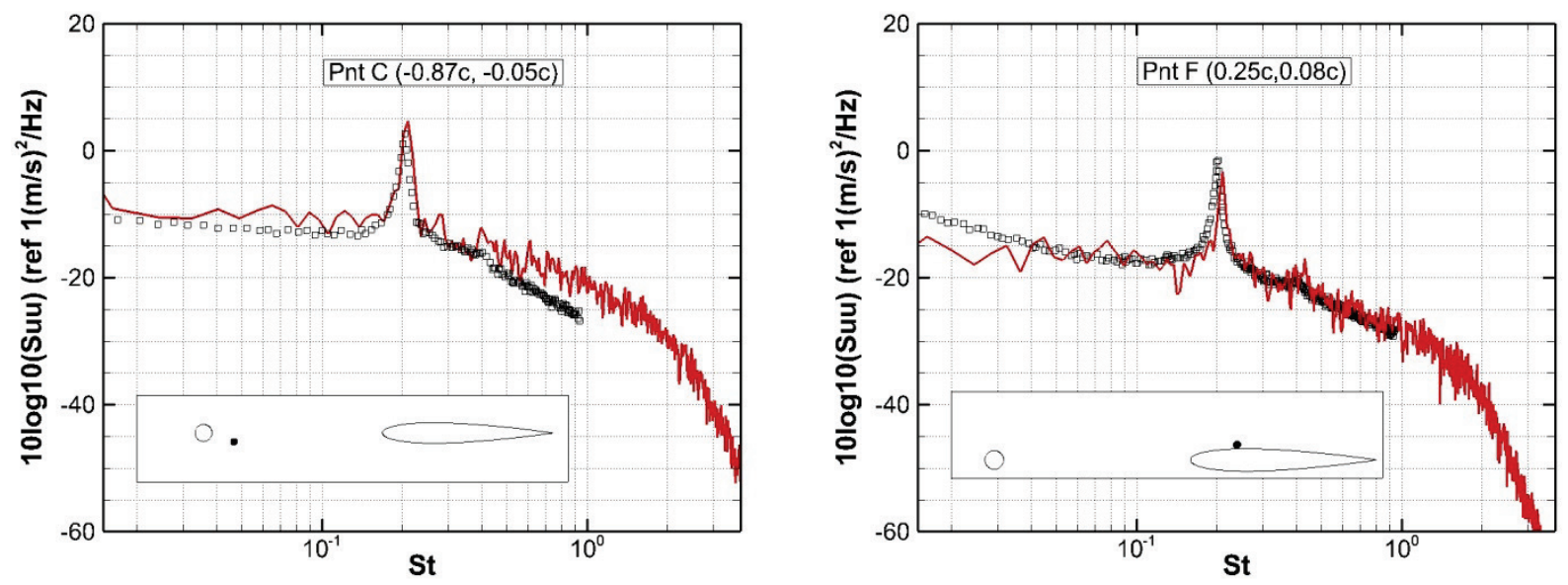

Figure 17. PSD of streamwise velocity fluctuations at six points.

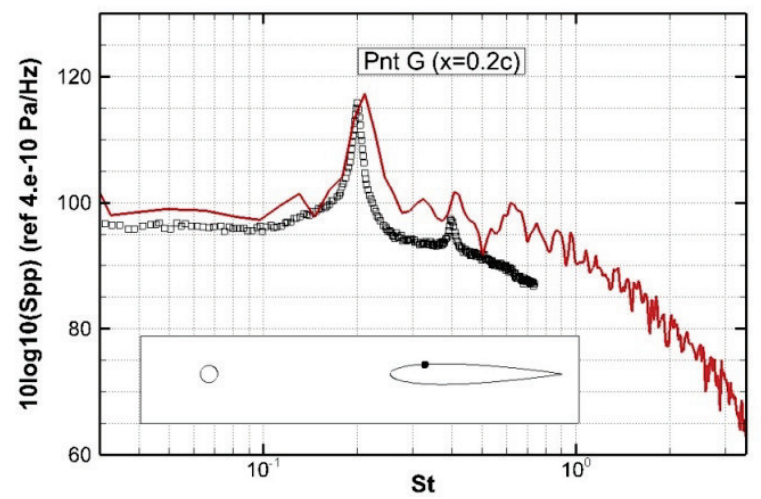

Figure 18. PSD of wall pressure fluctuations at $G$ point. Red line: Compt. Black square: Exp.

To obtain the far-field noise radiation, the time-domain formulation is solved with the advanced time approach $^{26}$. For low Mach number flows, the omission of quadrupole source has little influence on the noise radiation ${ }^{11}$. Therefore, the time accurate data is collected on the surface of both the rod and the airfoil. Besides, as mentioned in many studies ${ }^{16,24,25}$, the spanwise length in CFD is typically a small portion of the experimental spanwise length. In this paper, the CFD spanwise length is $0.3 c$ and experimental spanwise length is $3 c$. Hence, special treatments should be taken to correct the farfield noise results. The widely adopted Kato's correction ${ }^{27}$ is usually recommended. However, in this paper, the input data for FW-H equation are replicated 10 times in the spanwise direction. The PSD of pressure at three farfield locations is shown in Figure 19. The peak amplitude agrees with the experimental data well. At high frequencies, the sound pressure is slightly under-predicted. This is likely due to the ommision of the quadrupole source. Furthermore, Figure 20 gives the farfield directivity profile, which is also in excellent agreement with the experimental data. 

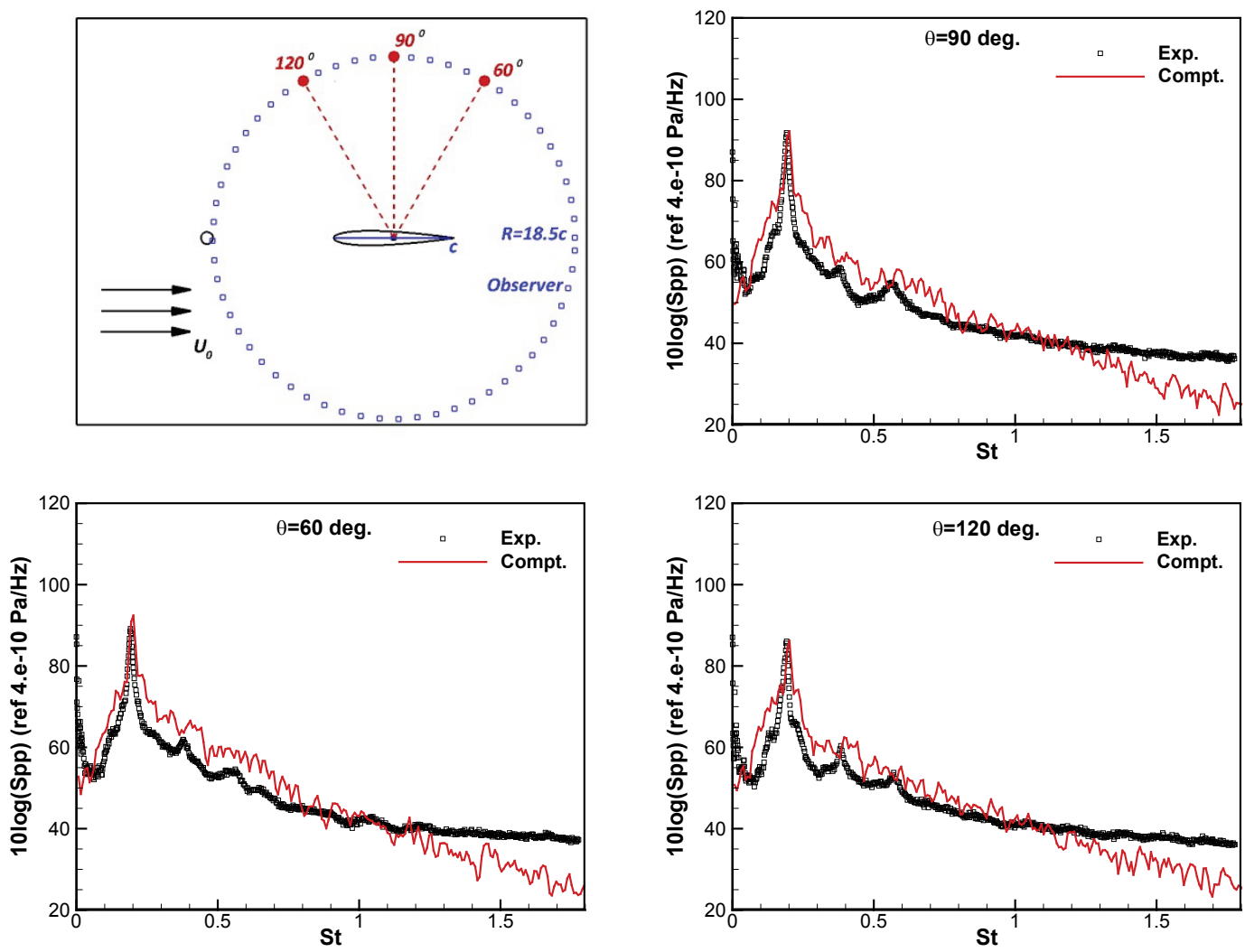

Figure 19. PSD of farfield pressure fluctuations at three observers on the circle with $R=18.5 \mathrm{c}$.

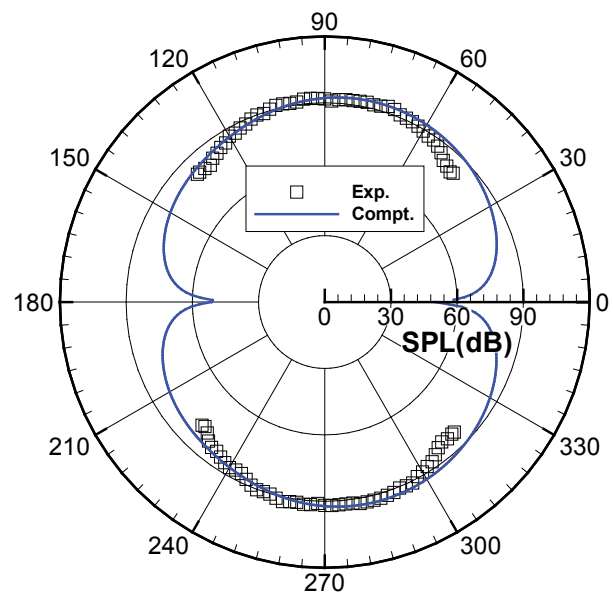

Figure 20. Directivity of PSD of farfield peak pressure at $R=18.5 \mathrm{c}$.

\section{Conclusions and future work}

The cell-centered finite difference method (CCFDM), due to its satisfaction of the geometric conservation law, is an efficient and robust method for numerical simulation with complicated configurations on a curvilinear structured grid. In this paper, further development of the method, by extending cell-centered finite difference method (CCFDM) for aeroacoustic problems has been investigated. This paper contains two parts.

The first part aims to develop appropriate schemes for direct noise calculation. Specifically, a class of low-dissipation and low-dispersion schemes within the framework of CCFDM is proposed. The benchmark cases 
from CAA workshops are used for validation and the results indicate that the new schemes are appropriate for long-time, long-distance wave propagation.

The second part of the paper investigates the feasibility of combining CCFDM with the FW-H equation for the far-field noise prediction. Two example cases are chosen to validate the frequency domain formulation and time domain formulation respectively. The noise radiated from 2D laminar flow past a NACA0012 airfoil is used to demonstrate that the convective effect should be taken into consideration if the Mach number is over 0.3. The broadband noise radiated from a 3D rod-airfoil configuration shows that CCFDM with time-domain formulation is an effective tool for far-field noise prediction.

The next step is to further study the potential of CCFDM combined with FW-H equation in high-speed turbulent flows.

\section{Acknowledgments}

This research is financially supported by 111 project of China (No. B17037) and China Scholarship Council (No. 201706290086).

\section{References}

${ }^{1}$ Lele S. K., "Compact finite difference schemes with spectral-like resolution." Journal of Computational Physics, Vol. 103, No.1, 1992, pp. 16-42.

${ }^{2}$ Kim J. W., "Optimized boundary compact finite difference schemes for computational aeroacoustics." Journal of Computational Physics, Vol. 225, No.1, 2007, pp. 995-1019.

${ }^{3}$ Thomas P. D. and Lombard C. K., "Geometric conservation law and its application to flow computations on moving grids." AIAA Journal, Vol. 17, No. 10, 1979, 1030-1037.

${ }^{4}$ Deng X. G., Mao M. L., Tu G. H., Liu H. Y. and Zhang H. X., "Geometric conservation law and applications to high-order finite difference schemes with stationary grids." Journal of Computational Physics, Vol. 230, No. 4, 2011, pp. 1100-1115.

${ }^{5}$ Deng X. G., Mao M. L., Tu G. H., Zhang H. X. and Zhang Y. F. "High-Order and High Accurate CFD Methods and Their Applications for Complex Grid Problems." Communications in Computational Physics, Vol. 11, No. 4, 2012, pp. 1081-1102.

${ }^{6}$ Liao F., Ye Z. Y. and Zhang L. X., "Extending geometric conservation law to cell-centered finite difference methods on stationary grids." Journal of Computational Physics, Vol. 284, 2015, pp. 419-433.

${ }^{7}$ Liao F. and Ye Z. Y., "Extending geometric conservation law to cell-centered finite difference methods on moving and deforming grids." Journal of Computational Physics, Vol. 303, 2015, pp. 212-221.

${ }^{8}$ Nonomura T. and Fujii K., "Effects of difference scheme type in high-order weighted compact non-linear schemes." Journal of Computational Physics, Vol. 228, No. 10, 2009, pp. 3533-3539

${ }^{9}$ Deng X. G., Jiang Y., Mao M. L., Liu H. Y., Li S. and Tu G. H., "A family of hybrid cell-edge and cell-node dissipative compact schemes satisfying geometric conservation law." Computers and Fluids, Vol. 116, No 15, 2015, pp. 29-45.

${ }^{10}$ Williams J. E. F. and Hawkings D. L., "Sound Generation by Turbulence and Surfaces in Arbitrary Motion." Philosophical Transactions of the Royal Society A: Mathematical, Physical and Engineering Sciences, Vol. 264, No. 1151, 1969, pp.321-342.

${ }^{11}$ Brentner K. S. and Farassat F., "Modeling aerodynamically generated sound of helicopter rotors." Progress in Aerospace Sciences, Vol. 39, No. 2-3, 2003, pp. 83-120.

${ }^{12}$ Ghorbaniasl G. and Lacor C., "A moving medium formulation for prediction of propeller noise at incidence." Journal of Sound and Vibration, Vol. 331, No. 1, 2012, pp.117-137.

${ }^{13}$ Lockard D. P., "An efficient, two-dimensional implementation of the Ffowcs Williams and Hawkings equation." Journal of Sound and Vibration, Vol. 229, No. 4, 2000, pp. 897-911.

${ }^{14}$ Gloerfelt X., Bailly C. and JuvÉ D., "Direct computation of the noise radiated by a subsonic cavity flow and application of integral methods." Journal of Sound and Vibration, Vol. 266, No. 1, 2003, pp.119-146.

${ }^{15}$ Wolf W. R. and Lele S. K., "Acoustic Analogy Formulations Accelerated by Fast Multipole Method for Two-Dimensional Aeroacoustic Problems.” AIAA Journal, Vol. 48, No. 10, 2015, pp. 2274-2285.

${ }^{16}$ Jacob M. C., Boudet J., Casalino D. and Michard M., "A rod-airfoil experiment as a benchmark for broadband noise modeling." Theoretical and Computational Fluid Dynamics, Vol. 19, No. 3, 2005, pp.171-196.

${ }^{17}$ Liu X. L., Zhang S. H., Zhang H. X. and Shu C. W., "A new class of central compact schemes with 
spectral-like resolution I: Linear schemes." Journal of Computational Physics, Vol. 248, No 1, 2013, pp. 235-256.

${ }^{18}$ Hardin J. C., Ristorcelli J. R. and Tam C. K. W., "ICASE/LaRC workshop on benchmark problems in computational aeroacoustics (CAA)." NASA-CP-3300, L-17497, NAS 1.55:3300, 1995.

${ }^{19}$ Kim J. W. and Lee D. J., "Fourth computational aeroacoustics (CAA) workshop on benchmark problems." NASA/CP-2004-212954, E-14393, 2004.

${ }^{20}$ Li X. L., Leng Y. and He Z. W., "Optimized sixth-order monotonicity-preserving scheme by nonlinear spectral analysis: optimized monotonicity-preserving scheme." International Journal for Numerical Methods in Fluids, Vol. 73, No. 6, 2013, pp. 560-577.

${ }^{21}$ Liao F., Jin Y., Cai J. S. and Ye Z. Y., "Iterative implicit boundary treatment to DDADI/D3ADI method for Navier-Stokes equations." (To be submitted)

${ }^{22}$ Spalart P. R., Deck S., Shur M. L., Squires K. D., Strelets M. Kh. and Travin A., "A New Version of Detached-eddy Simulation, Resistant to Ambiguous Grid Densities." Theoretical and Computational Fluid Dynamics, Vol. 20, No. 3, 2006, 181-195.

${ }^{23}$ Apelt C. J. and West G. S., "The Effects of Wake Splitter Plates on Bluff-Body Flow in the Range $104<\operatorname{Re}<$ 5×104. Part 2." Journal of Fluid Mechanics, Vol. 71, No. 1, 1975, pp.145-160.

${ }^{24}$ Giret J. C., Sengissen A., Moreau S., Sanjose M. and Jouhaud J. C., "Noise source analysis of a rod-airfoil configuration using unstructured large eddy simulation." AIAA Journal, Vol. 53, No. 4, pp. 1062-1077

${ }^{25}$ Agrawal B. R. and Sharma A., "Aerodynamic Noise Prediction for a Rod-Airfoil Configuration using Large Eddy Simulations." 20th AIAA/CEAS Aeroacoustics Conference. AIAA paper, 2014.

${ }^{26}$ Casalino D., "An advanced time approach for acoustic analogy predictions." Journal of Sound and Vibration, Vol. 261, No. 4, 2003, pp. 583-612.

${ }^{27}$ Kato C. and Ikegawa M., "Large Eddy Simulation of Unsteady Turbulent Wake of a Circular Cylinder using the Finite Element Method." Advances in Numerical Simulation of Turbulent Flows, Vol. 1, 1991, pp. 49-56. 